\title{
Wakes in a Collisional Quark-Gluon Plasma
}

\author{
Purnendu Chakraborty * Munshi Golam Mustafa. $]^{\dagger}$ and Rajarshi Ray $\#$ \\ Theory Division, Saha Institute of Nuclear Physics, \\ 1/AF Bidhannagar, Kolkata 700064. \\ Markus H. Thomas \\ Max-Planck-Institut für extraterrestrische Physik, \\ P.O. Box 1312, 85741 Garching, Germany
}

\begin{abstract}
Wakes created by a parton moving through a static and infinitely extended quark-gluon plasma are considered. In contrast to former investigations collisions within the quark-gluon plasma are taken into account using a transport theoretical approach (Boltzmann equation) with a BhatnagarGross-Krook (BGK) collision term. Within this model it is shown that the wake structure changes significantly compared to the collisionless case.
\end{abstract}

PACS numbers: 12.38.Mh,25.75.Nq,52.25.Dg,52.25.Fi

Keywords: quark-gluon plasma, collisional plasmas, wakes

*Electronic address: purnendu.chakraborty@saha.ac.in

${ }^{\dagger}$ Electronic address: musnhigolam.mustafa@saha.ac.in

${ }^{\ddagger}$ Electronic address: rajarshi.ray@saha.ac.in

$\S$ Electronic address: thoma@mpe.mpg.de 
In ultrarelativistic heavy-ion experiments (SPS, RHIC) indications have been found for a temporary formation of a strongly coupled quark-gluon plasma (QGP) phase in the fireball created in nucleus-nucleus collisions (see e.g. Ref.[1]). The main problem of this programme is to find clear signatures for the presence of a short-living QGP phase in this fireball. One promising class of signatures are hard probes, in particular high-energy partons of a few $\mathrm{GeV}$ or more, i.e. with an energy much higher than the temperature of the QGP. Energetic partons with a large transverse momentum, produced in initial hard parton collisions before the formation of an equilibrated fireball, propagate through the fireball. In this process they will lose energy, which depends on the state of the fireball (QPG or hadronic matter). Energetic partons manifest themselves at jets arriving in the detectors. The amount of jet quenching due to the propagation of the leading parton of the jet through the fireball can therefore serve as a signature for the QGP formation [2, 3]. A strong jet quenching and suppression of high $p_{T}$ hadron spectra have been observed at RHIC indicating the presence of a QGP phase [4]. Another phenomenon related to the propagation of a high-energy parton through the QGP are wakes and Mach cones which may be observable as conical flow and shock waves in particle correlations [5, 6, 6, 8, [9, 10].

In electromagnetic plasmas wakes are a well known phenomena. Theoretically they can be investigated by considering transport equations. In the collisionless case they follow from the dielectric functions by solving the Vlasov equation together with the Poisson equation. It has been shown in this way that wakes are also created in a QGP, leading eventually to attractive potentials and Mach cones as well known in plasma physics (see below). Attractive potentials between partons might lead to bound states and may modify the $J / \psi$ suppression pattern [9]. In the static case, i.e. for a parton at rest, the wake potential reduces to the usual Yukawa potential describing Debye screening [12].

Here we want to extend these investigations by taking into account collisions in the QGP. For this purpose we start from the Boltzmann equation approximating the collision term by the BGK description [13], allowing an analytic expression for the dielectric functions [14, 15]. In this approach the collision term is replaced by a momentum independent collision rate which we take as a parameter. This model has been used to study dispersion relations [14] and instabilities [16] in a collisional QGP. Whereas the changes in the case of the dispersion relations are marginal - however the longitudinal plasma modes cross the light cone in a collisional plasma -, instabilities can be suppressed efficiently due to collisions. In the case 
of complex plasmas, i.e., low-temperature discharge plasmas with microparticles such as dust grains [17], wake potentials due to ion flow, leading to an attractive force between the negatively charged microparticles, are investigated intensively (see e.g. [18]). Also collisions between the ions and the neutral gas have been taken into account in this case and have been shown to truncate the wakes effectively [19]. In general the collisions modify the wake potential by decelerating the ions. Therefore the positive ion cloud, responsible for the attractive part of the wake potential, is concentrated nearer to the dust grain, causing a narrower but deeper potential well.

The longitudinal and transverse dielectric functions of a collisional QGP within the BGK approach are given by [14],

$$
\begin{aligned}
& \epsilon_{l}(\omega, k)=1+\frac{m_{D}^{2}}{k^{2}}\left(1-\frac{\omega+i \nu}{2 k} \ln \frac{\omega+i \nu+k}{\omega+i \nu-k}\right)\left(1-\frac{i \nu}{2 k} \ln \frac{\omega+i \nu+k}{\omega+i \nu-k}\right)^{-1} \\
& \epsilon_{t}(\omega, k)=1-\frac{m_{D}^{2}}{2 \omega(\omega+i \nu)}\left\{1+\left[\frac{(\omega+i \nu)^{2}}{k^{2}}-1\right]\left(1-\frac{\omega+i \nu}{2 k} \ln \frac{\omega+i \nu+k}{\omega+i \nu-k}\right)\right\}
\end{aligned}
$$

where $\nu$ is the collision rate and $m_{D}=\sqrt{1+n_{f} / 6} g T$ the Debye screening mass. Here $n_{f}$ is the number of quark flavors in the QGP. From the dielectric functions the dispersion relations follow [14], e.g. the longitudinal mode (plasmon) from $\epsilon_{l}(\omega, k)=0$. Note that $\omega$ has a real and imaginary part here.

The induced charge density by a parton with color charge $Q^{a}$ propagating through the QGP with velocity $v$ follows from [9]

$$
\rho_{\text {ind }}^{a}(t, \overrightarrow{\mathbf{r}})=2 \pi Q^{a} \int \frac{d^{3} k}{(2 \pi)^{3}} \int \frac{d \omega}{2 \pi} \exp [i(\vec{k} \cdot \vec{r}-\omega t)]\left[\frac{1}{\epsilon_{l}(\omega, k)}-1\right] \delta(\omega-\vec{k} \cdot \vec{v}) .
$$

Due to the delta function $\omega$ is restricted here to real values and is always in the space-like region for $0<v<1$.

The induced charge density using cylindrical coordinates can be written as [20]

$$
\begin{aligned}
\rho_{\text {ind }}^{a}(\rho, z, t) & =\frac{Q^{a}}{2 \pi^{2} v} \int_{0}^{\infty} d \kappa \kappa J_{0}(\kappa \rho) \int_{0}^{\infty} d \omega\left\{\cos \left[\omega\left(\frac{z}{v}-t\right)\right]\left(\frac{\operatorname{Re} \epsilon_{l}}{\Delta}-1\right)\right. \\
& \left.+\sin \left[\omega\left(\frac{z}{v}-t\right)\right] \frac{\operatorname{Im} \epsilon_{l}}{\Delta}\right\},
\end{aligned}
$$

where $J_{0}$ is the Bessel function, $\Delta=\left[\operatorname{Re} \epsilon_{l}\right]^{2}+\left[\operatorname{Im} \epsilon_{l}\right]^{2}$, and $\kappa=\sqrt{k^{2}-\omega^{2} / v^{2}}$.

The real and imaginary parts of the dielectric functions read

$$
\operatorname{Re} \epsilon_{l}=1+\frac{m_{D}^{2}}{4 k^{4} f(\omega, k, \nu)}\left[4 k^{2}+\nu^{2}\left(\theta^{2}+\ln ^{2} R\right)-2 k(\omega \ln R+2 \nu \theta)\right],
$$




$$
\operatorname{Im} \epsilon_{l}=\frac{m_{D}^{2} \omega}{4 k^{4} f(\omega, k, \nu)}\left[2 k \theta-\nu\left(\ln ^{2} R+\theta^{2}\right)\right],
$$

where

$$
\begin{aligned}
& R=\frac{\sqrt{\left(\omega^{2}-k^{2}+\nu^{2}\right)^{2}+4 k^{2} \nu^{2}}}{(\omega-k)^{2}+\nu^{2}} \\
& \theta=\arccos \frac{\omega^{2}-k^{2}+\nu^{2}}{\sqrt{\left(\omega^{2}-k^{2}+\nu^{2}\right)^{2}+4 k^{2} \nu^{2}}},
\end{aligned}
$$

and

$$
f(\omega, k, \nu)=\left(1-\frac{\nu \theta}{2 k}\right)^{2}+\frac{\nu^{2} \ln ^{2} R}{4 k^{2}} .
$$

Solving (3) together with (5) numerically [21] leads to the induced charge densities shown in Figs.1 and 2. The induced densities are proportional to $m_{D}^{3}$ and are scaled with $m_{D}$ as well as with the color charge $Q^{a}$ of the moving parton. Assuming for example $n_{f}=2, g=2$ (corresponding to $\alpha_{s}=1 / \pi$ ), and $T=250 \mathrm{MeV}$, we get a typical value of $m_{D}=580 \mathrm{MeV}$. In Fig. 1 the parton velocity is $v=0.55 c$ and $v=0.99 c$ in Fig.2. The left panels show the 3 dimensional plots for the choice $\nu=0$ to $\nu=0.8 m_{D}$ [22]. The right panels show the contour plots of the induced charge density. One observes a clear modification of the induced charge density due to the collision rate. The general structure of the wakes, corresponding to a modified screening, a cone-like structure, and wave excitation has been discussed in detail in Ref.[9] in the collisionless case. However, in the case of collisions these structures are smeared out and less pronounced for increasing $\nu$. We interpret this as the fact that the collisions, leading to thermalization, reduce the anisotropy caused by the perturbation of the moving charge in the QGP.

The wake potential is given by (see appendix)

$$
\Phi^{a}(\vec{r}, t ; \vec{v})=\frac{Q^{a}}{2 \pi^{2}} \int d^{3} k \frac{e^{-i \vec{k} \cdot(\vec{r}-\vec{v} t)}}{k^{2} \epsilon_{l}(\omega=\vec{v} \cdot \vec{k}, k)} .
$$

It is of the same form as in the collisionless case, where it can be shown that in cylindrical coordinates it reduces to [9]

$$
\begin{aligned}
\Phi^{a}(\rho, z, t)= & \frac{2 Q^{a}}{\pi v} \int_{0}^{\infty} d \kappa \kappa J_{0}(\kappa \rho) \int_{0}^{\infty} d \omega \frac{1}{k^{2} \Delta}\left\{\cos \left[\omega\left(\frac{z}{v}-t\right)\right] \operatorname{Re} \epsilon_{l}\right. \\
& \left.+\sin \left[\omega\left(\frac{z}{v}-t\right)\right] \operatorname{Im} \epsilon_{l}\right\} .
\end{aligned}
$$

In Figs. 3 and 4 the wake potentials scaled with respect to $m_{D}$ are shown for a parton with a given color charge $Q^{a}$ for velocities $v=0.55 c$ and $v=0.99 c$ and various values 
for the collision rate from $\nu=0$ to $\nu=0.8 m_{D}$. It can be seen that collisions modify the strength and the structure of the wake potential significantly. Again, as in the case of the induced charge density the potential structure is washed out due to the collisions between the partons in the QGP. However, the potential becomes also stronger at finite collision rates, indicating that collisions in the QGP can enhance the attractive and repulsive parts of the interaction potential of a fast parton as in the case of non-relativistic plasmas. In particular, the attractive part behind the particle becomes deeper which might lead to an even stronger binding of diquark states propagating through the QGP as discussed in Ref.[9]. (Note that the induced charge density and wake potential diverge for point-like particles at the origin as in the static case corresponding to a Yukawa potential.)

In conclusion, we have applied the BGK approach in order to study the influence of collisions on the wake formation in the QGP. Clearly, this approach allows only qualitative statements. A consistent QCD approach, in which for example the collision rate are momentum dependent, is beyond the scope of this work. Keeping this in mind, we regard the (momentum independent) collision rate in our model as a free parameter. Anyway, our model demonstrates clearly that collisions in the QGP lead to a significant modification of wakes created by fast partons compared to the collisionless case. In particular, the wake structure is less pronounced. Hence possible observable effects such as the conical flow and wave excitations will be reduced. On the other hand, the attractive potential well becomes deeper. Similar effects of collisions have been observed in electromagnetic plasmas.

Acknowledgments We would like to thank M. Strickland for helpful discussions. M.H.T. would like to thank A. Ivlev for information on wakes in complex plasmas. MGM and RR are also thankful to S. Mallik for useful discussions.

\section{APPENDIX}

Here we will explicitly derive equation (6) for the wake potential in the case of collisions. For the collisionless case it follows from a combination of the linearized Vlasov equation and the Poisson equation. Here we start from the kinetic equation with a BGK-collision term. The derivation from the equilibrium distribution function $f_{0}(p)$ is then given by (see (14) in 
Ref.[14])

$$
\delta f(\vec{p}, \vec{r}, t)=\left[-i q \vec{E} \cdot \frac{\partial f_{0}(p)}{\partial \vec{p}}+i \nu \eta f_{0}(p)\right] D^{-1}
$$

where $q$ is the charge of the plasma particles and $D:=\omega+i \nu-\vec{k} \cdot \vec{v}$ with $\vec{v}=\vec{p} / p$ in the ultrarelativistic case $(v=1)$.

The quantity $\eta$ is given by

$$
\eta=\frac{-i q}{N_{0}} \int d p \frac{(\vec{E} \cdot \vec{p})}{p} \frac{\partial f_{0}(p)}{\partial p} D^{-1}\left[1-\frac{i \nu}{N_{0}} \int d p f_{0}(p) D^{-1}\right]^{-1}
$$

with the equilibrium particle number density $N_{0}$ and $d p=d^{3} p /(2 \pi)^{3}$.

The Poisson equation for the potential $\Phi$ of a point charge $Q$ with velocity $\vec{v}$, where we drop the color index $a$ for convenience, in the plasma reads

$$
\Delta \Phi(\vec{r}, t ; \vec{v})=-4 \pi Q \delta(\vec{r}-\vec{v} t)-\rho(\vec{r}, t)
$$

where $\rho=q \int d p \delta f$ is the induced charge density in the plasma. In momentum space it reads

$$
k^{2} \Phi(\omega, \vec{k}, \vec{p})=8 \pi^{2} Q \delta(\omega-\vec{v} \cdot \vec{k})+q \int d p \delta f(\omega, \vec{k}, \vec{p}) .
$$

Using $\vec{E}=-i \vec{k} \Phi$ in momentum space and $\partial f_{0}(p) / \partial \vec{p}=\vec{v} \partial f_{0}(p) / \partial p$ we can write the Poisson equation by combining (11) with (8) and (9) as

$$
k^{2} \Phi(\omega, \vec{k}, \vec{p})=8 \pi^{2} Q \delta(\omega-\vec{v} \cdot \vec{k})+k^{2}(1-\chi) \Phi
$$

where

$$
\chi(\omega, k)=1+\frac{q^{2}}{k^{2}} \int d p(\vec{v} \cdot \vec{k}) \frac{\partial f_{0}}{\partial p} D^{-1}-\frac{i \nu q \eta}{k^{2} \Phi} \int d p f_{0} D^{-1} .
$$

Solving (12) for $\Phi$ and Fourier transforming it we obtain the wake potential

$$
\Phi(\vec{r}, t ; \vec{v})=\frac{Q}{2 \pi^{2}} \int d^{3} k \frac{e^{-i \vec{k}(\vec{r}-\vec{v} t)}}{k^{2} \chi(\omega=\vec{v} \cdot \vec{k})} .
$$

Comparing $\chi$ with the longitudinal dielectric function in the BGK approximation (see (17) and (18) in [14]),

$$
\epsilon_{l}(\omega, k)=1+\frac{q^{2}}{\omega k^{2}} \int d p(\vec{v} \cdot \vec{k})^{2} \frac{\partial f_{0}}{\partial p} D^{-1}-\frac{i \nu q \eta}{\omega k^{2} \Phi} \int d p(\vec{v} \cdot \vec{k}) f_{0} D^{-1}
$$

we find $\chi(\omega=\vec{v} \cdot \vec{k})=\epsilon_{l}(\omega=\vec{v} \cdot \vec{k})$. Hence (14) coincides with (6) and has the same form as in the collisionless case [9]. 
This result can also be derived more generally, i.e. in an homogeneous and isotropic plasma with and without collisions, assuming linear response theory for the induced charge

density [9], $\rho_{\text {ind }}=\left(1 / \epsilon_{l}-1\right) \rho_{\text {ext }}$, where the total charge density $\rho_{\text {tot }}=\rho_{\text {ext }}+\rho_{\text {ind }}$, and using the Poisson equation $\Phi=4 \pi \rho_{\text {ext }} /\left(k^{2} \epsilon_{l}\right)$ with $\rho_{\text {ext }}=2 \pi Q \delta(\omega-\vec{v} \cdot \vec{k})$ after Fourier transformation.

[1] Ullrich T 2007 Nucl. Phys. A 783 1c

[2] Plümer M and Gyulassy M 1990 Phys. Lett. B 243342

[3] Baier R, Schiff D, Zakharov B G 2000 Ann. Rev. Nucl. Part. Sci. 5037

[4] Adams et al. 2003 Phys. Rev. Lett. 91 172302; Adler S S et al. 2006 Phys. Rev. Lett. 96202301

[5] Stöcker H 2005 Nucl. Phys. A 750121

[6] Ruppert J and Müller B 2005 Phys. Lett. B 618123

[7] Satarov L M, Stöcker H, Mishustin I N 2005 Phys.Lett. B 62764

[8] Casalderrey-Solana J, Shuryak E V, Teaney D 2006 Nucl.Phys. A 774577

[9] Chakraborty P, Mustafa M G and Thoma M H 2006 Phys. Rev. D 74094002

[10] The wakes discussed here are associated with the collisional energy loss only not with the radiative [3]. However, the latter might also produce a large-angle radiation of gluons in addition to the one by Mach cones [11].

[11] Vitev I (2005) Phys. Lett. B 63078.

[12] Mustafa M G, Thoma M H and Chakraborty P 2005 Phys. Rev. C 71017901

[13] Bhatnagar P L, Gross E P and Krook M 1954 Phys. Rev. 94511

[14] Carrington M E, Fugleberg T, Pickering D and Thoma M H 2004 Can. J. Phys. 82671

[15] In Ref.[14] the BGK-approach has been derived for the QED case. However, since the Vlasov aproach for QED and QCD is the same (apart from color and flavor factors), this approach can be used also for QCD at least as a model for taking collisions into account (see also Ref.[16]).

[16] Schenke B, Strickland M, Greiner C and Thoma M H 2006, Phys. Rev. D 73125004

[17] Fortov V E, Ivlev A V, Khrapak S A, Khrapak A G and Morfill G E 2005 Phys. Rep 4211

[18] Bashkirov A G 2004 Phys. Rev. E 69046410

[19] Lampe M, Joyce G, Ganguli G, and Gavrishchaka V 2000 Phys. Plasmas 73851

[20] A typographical error in the factor $Q^{a} /(2 \pi)^{2} v$ in eq.(35) of Ref. [9] is now corrected here as 
$Q^{a} / 2 \pi^{2} v$

[21] We restrict to the physical Riemann sheet, i.e. $\ln z=\ln |z|+i \theta+2 i n \pi$ with $n=0$.

[22] The collision rate in the QGP is momentum dependent and of the order of $g^{2}$ for color and $g^{4}$ for momentum exchange [23]. However, since $g$ is of the order of one in realistic situations, the choice of $\nu$ between 0 and $m_{D}$ is reasonable.

[23] Thoma M H 1994 Phys. Rev. D 49451 

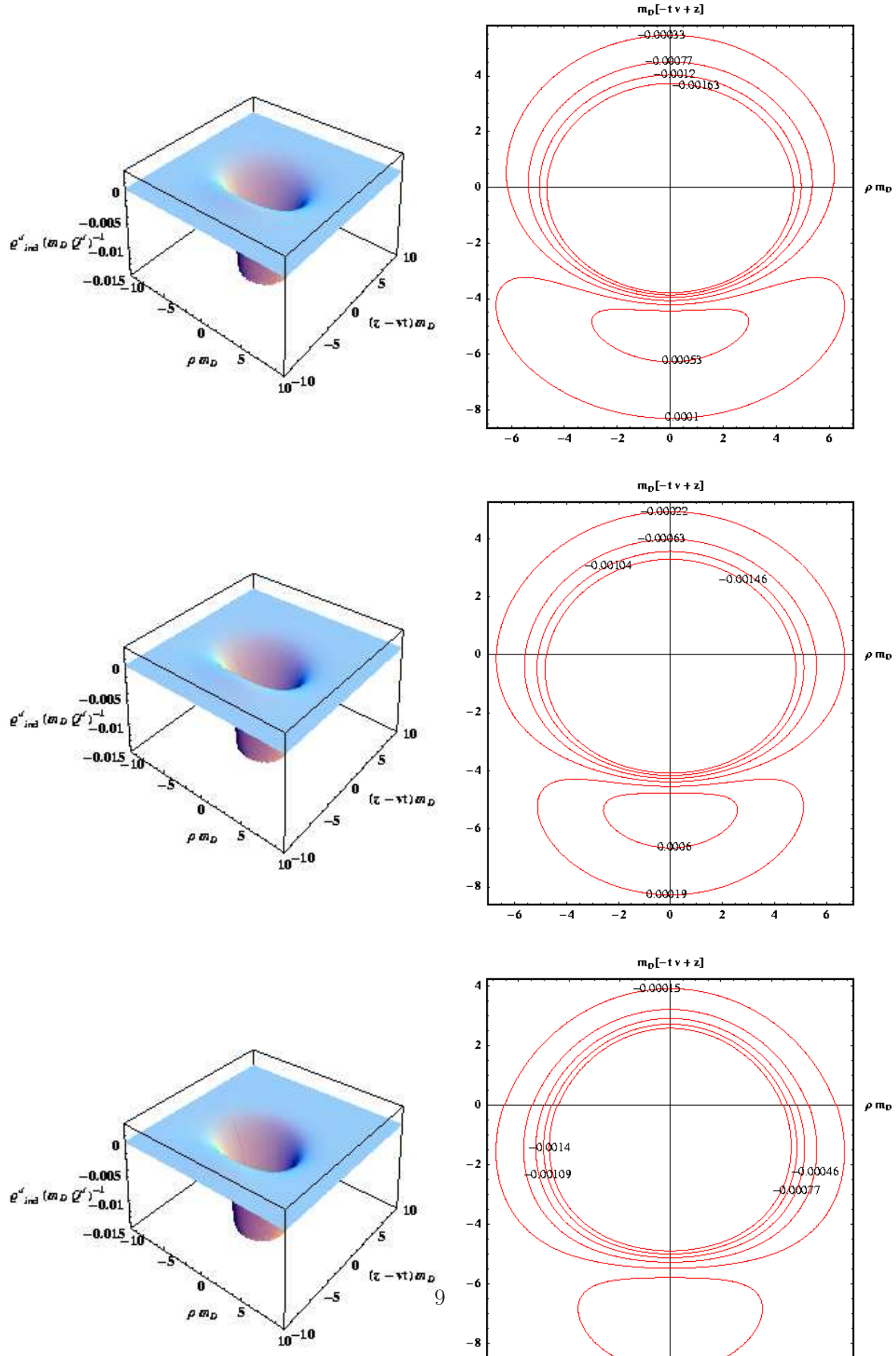

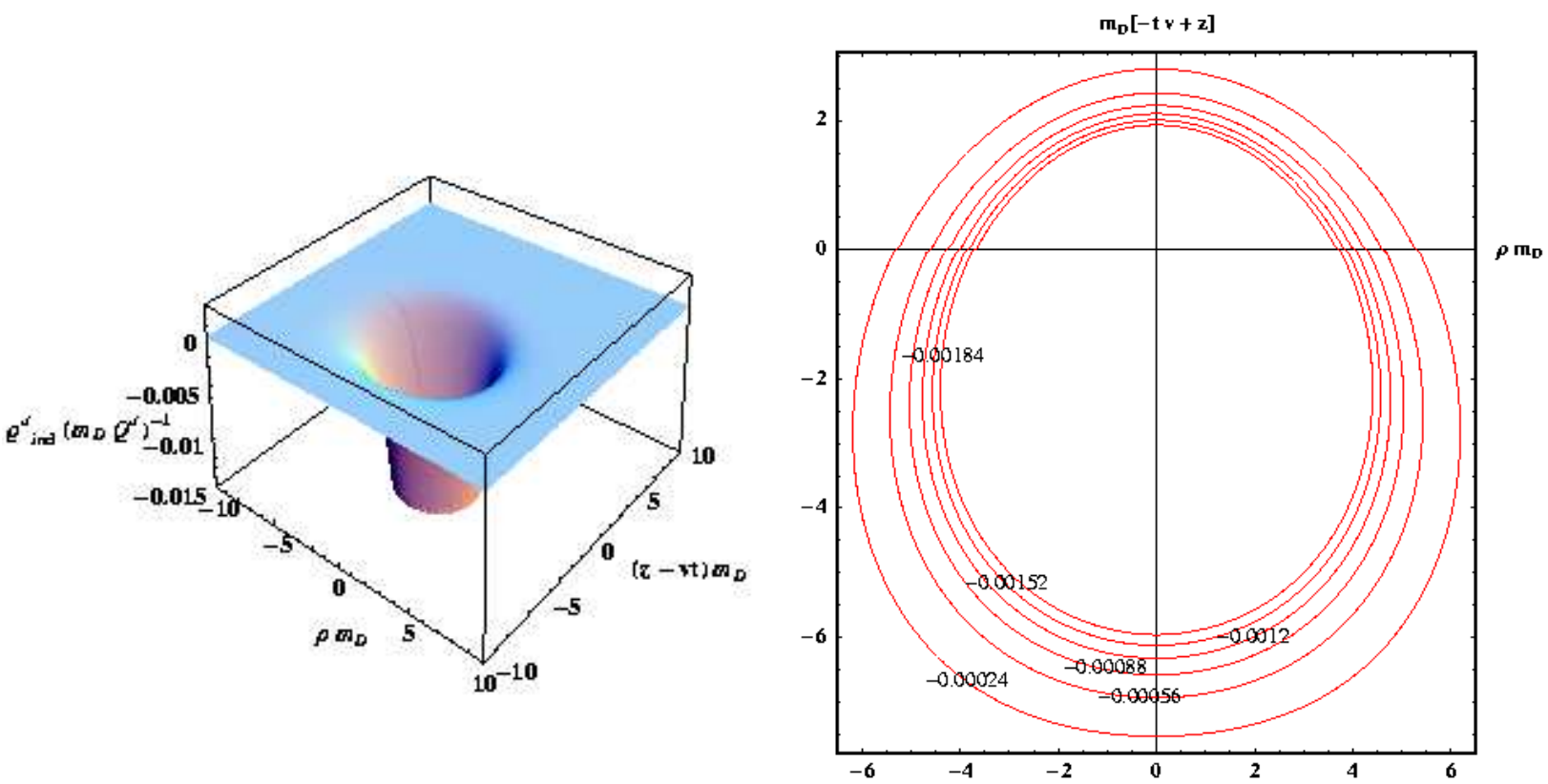

FIG. 1: Left panel: Spatial distribution of the induced charge density for a parton moving with velocity $v=.55 c$ with collisions for $\left(\nu=0,0.2 m_{D}, 0.5 m_{D}, 0.8 m_{D}\right)$ (Top to Bottom). Right panel: These plots show the corresponding contour plots 

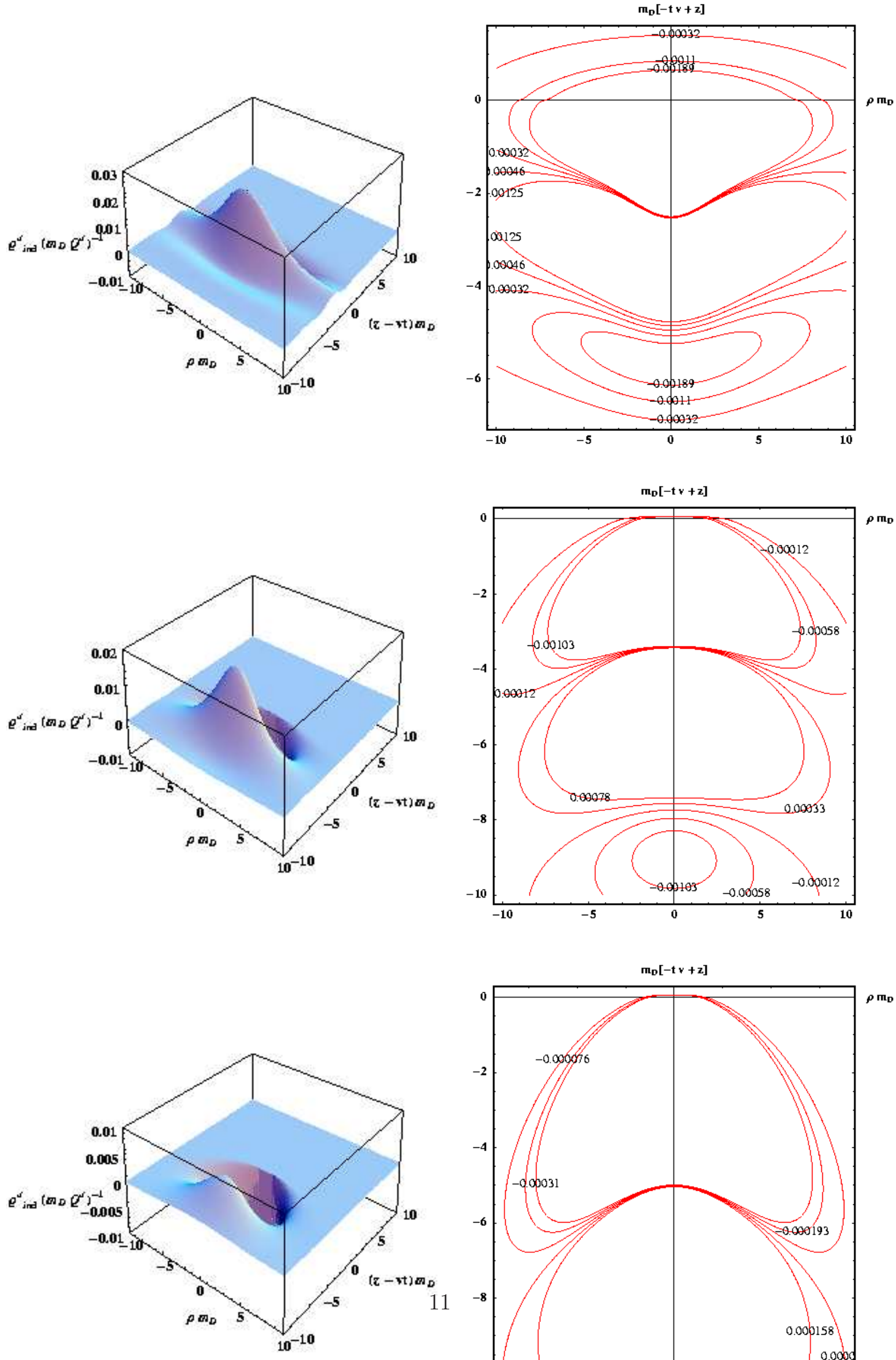

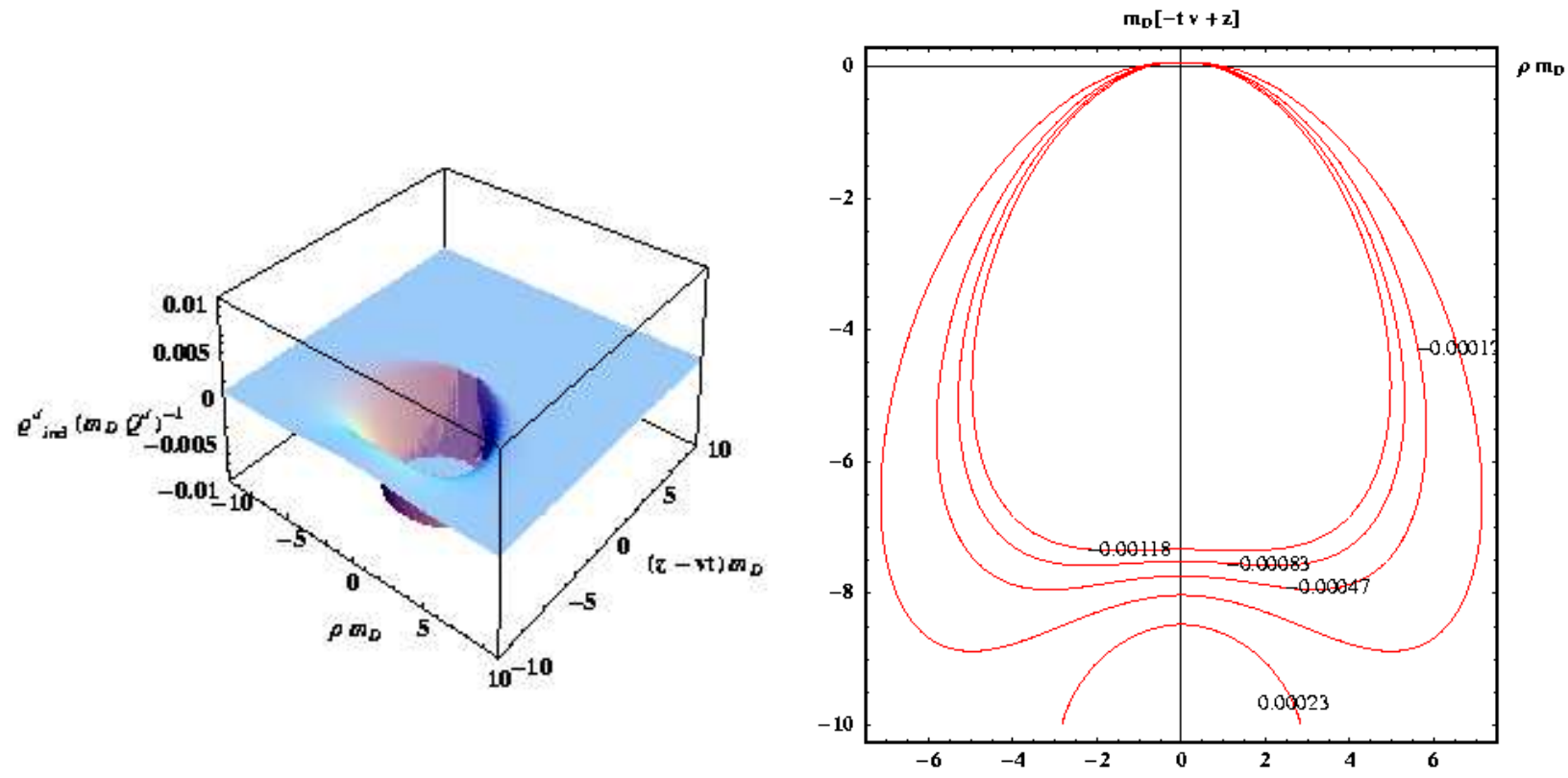

FIG. 2: Left panel: Spatial distribution of the induced charge density for a parton moving with velocity $v=.99 c$ with collisions for $\left(\nu=0,0.2 m_{D}, 0.5 m_{D}, 0.8 m_{D}\right)$ (Top to Bottom). Right panel: These plots show the corresponding contour plots 

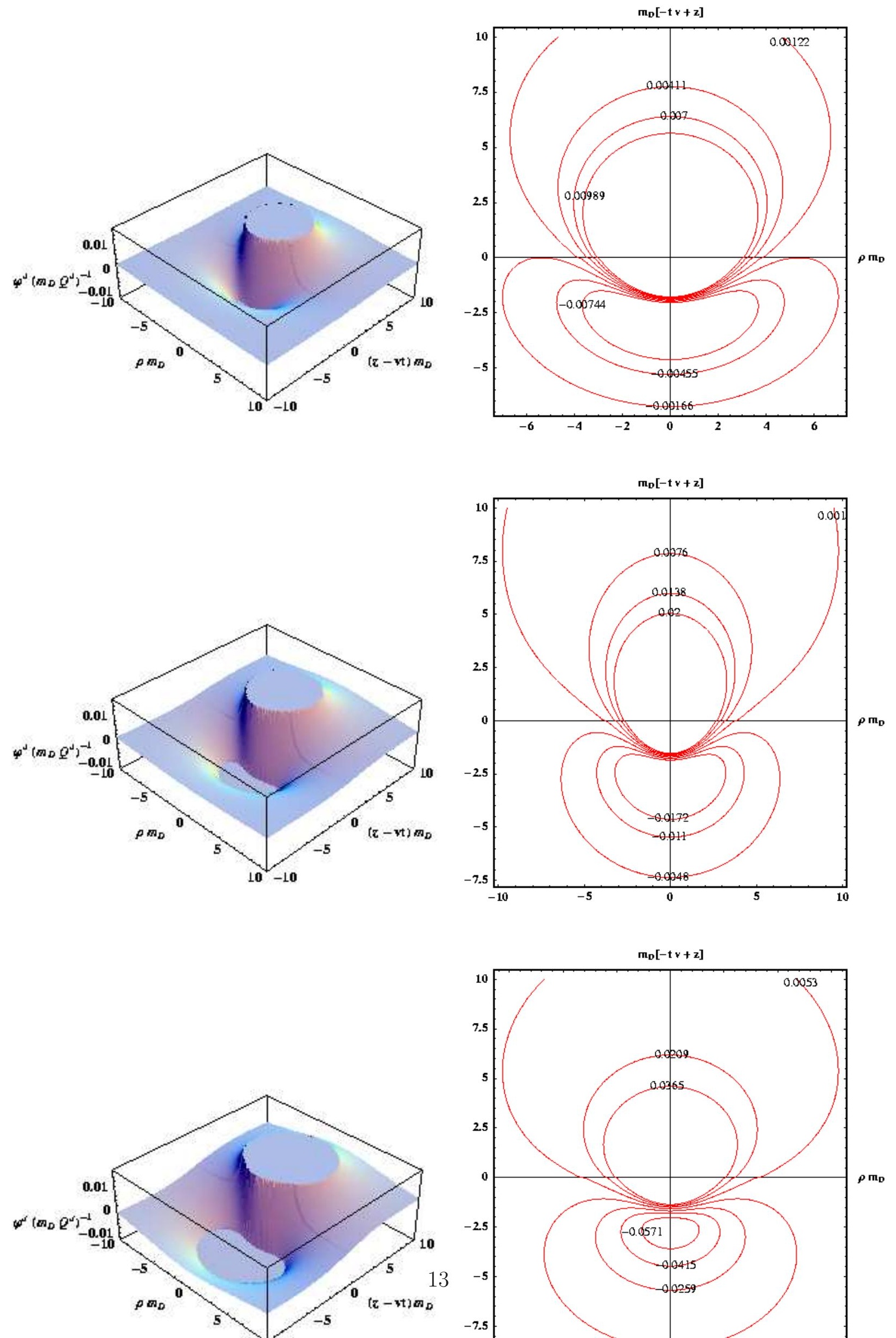

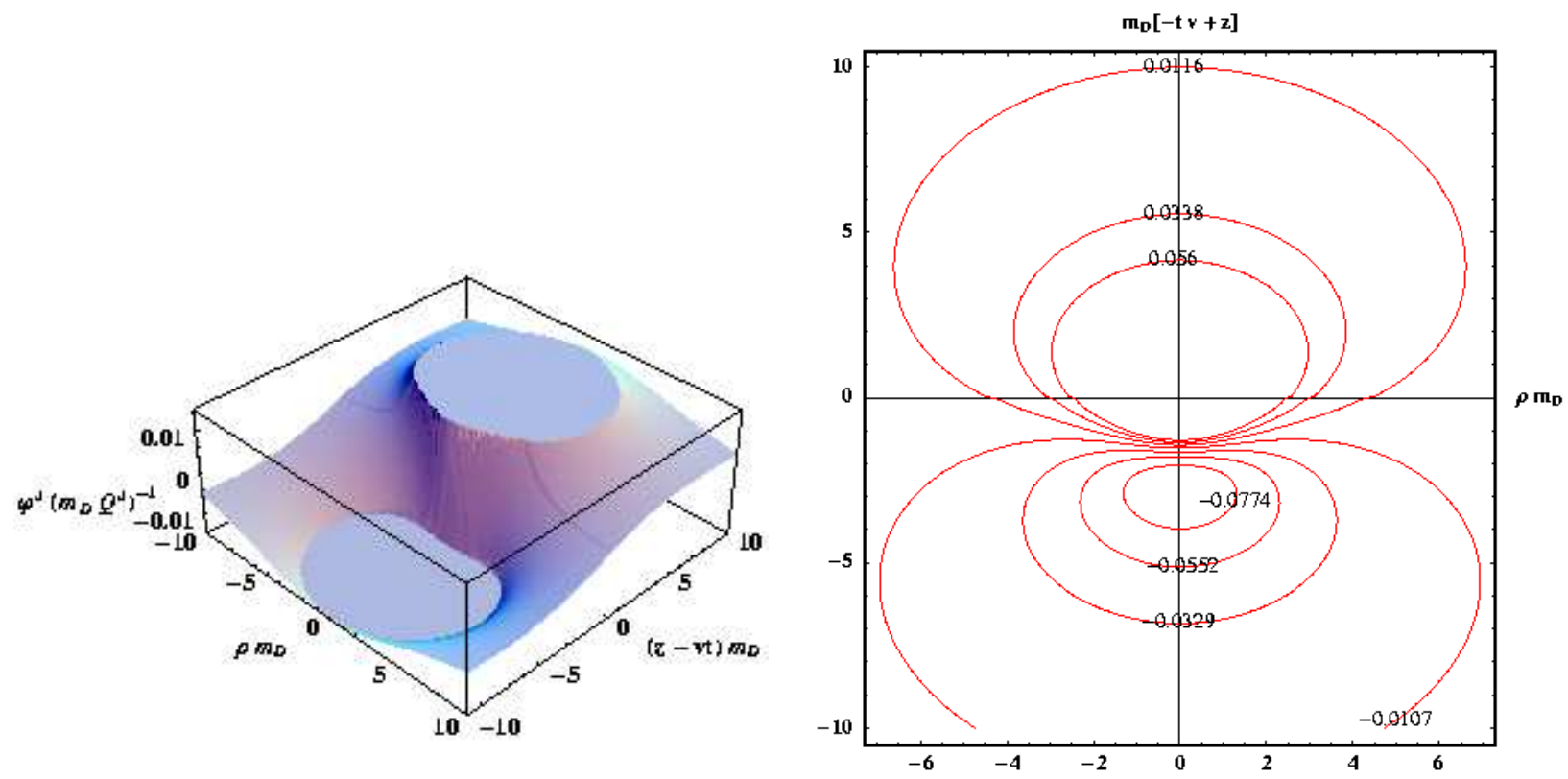

FIG. 3: Left panel: Spatial distribution of the scaled wake potentials for a parton moving with velocity $v=.55 c$ with collisions for $\left(\nu=0,0.2 m_{D}, 0.5 m_{D}, 0.8 m_{D}\right.$ (top to bottom panel). Right panel: These plots show the corresponding equipotential lines. 

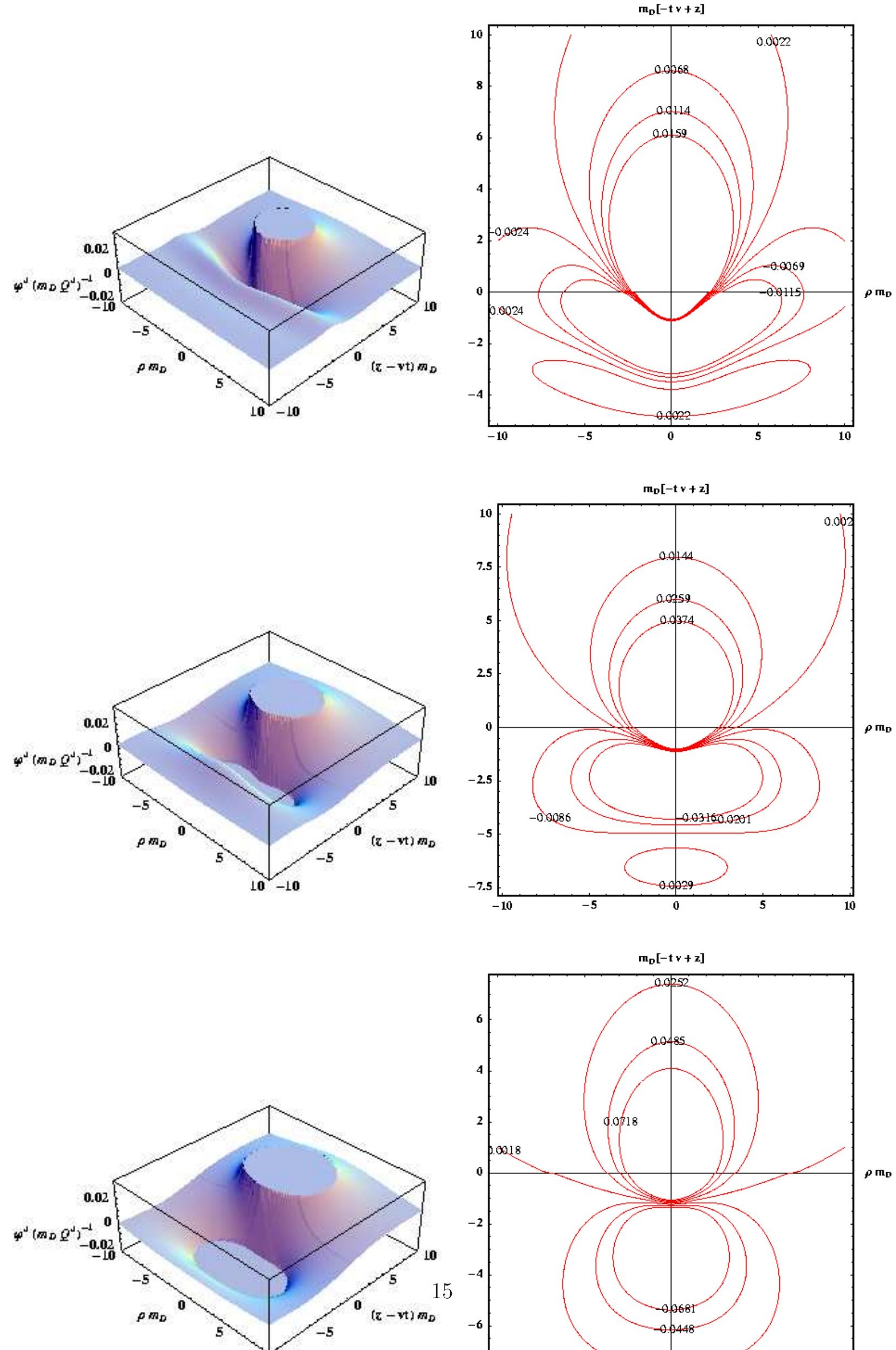

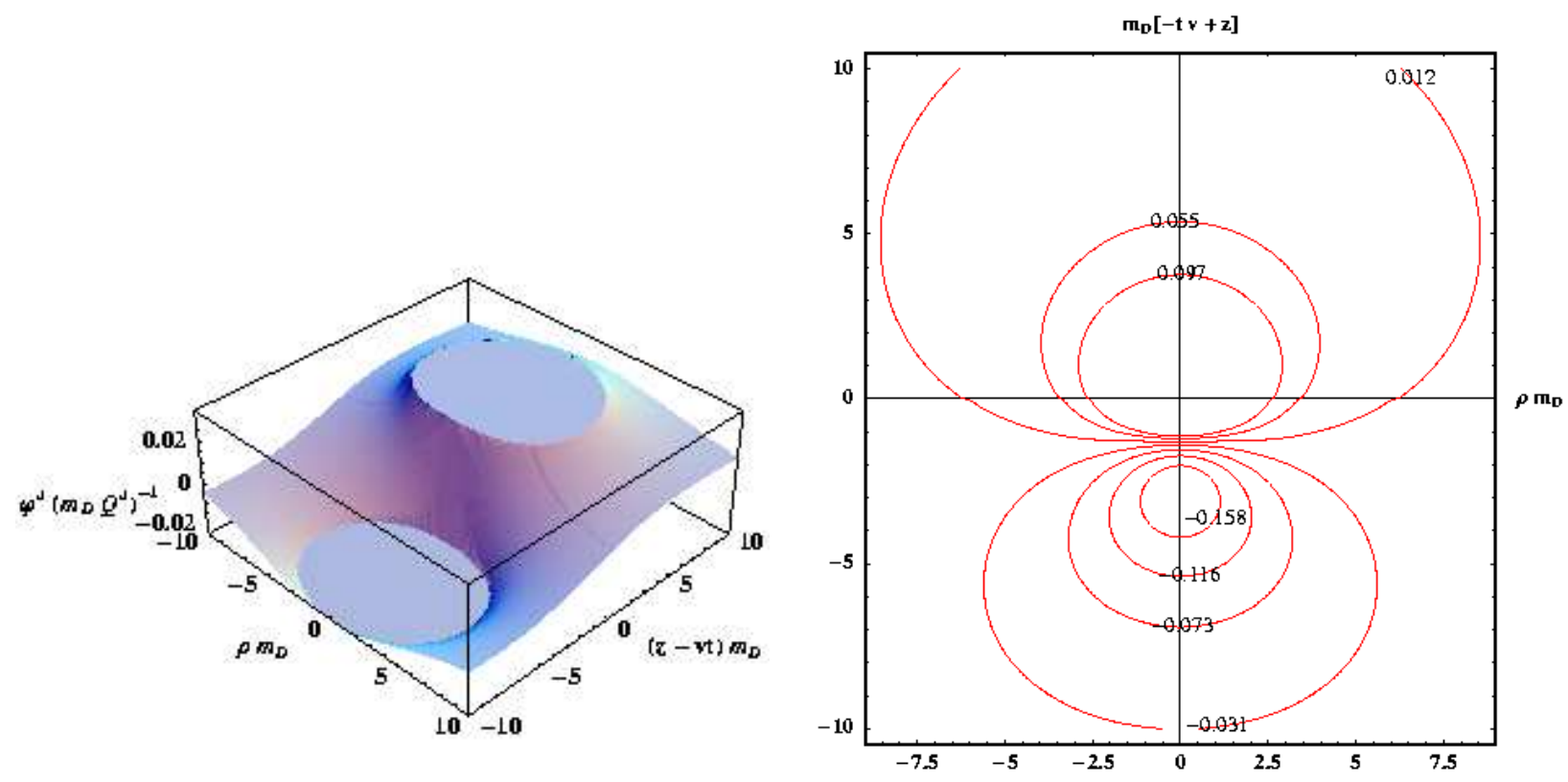

FIG. 4: Left panel: Spatial distribution of the scaled wake potentials for a parton moving with velocity $v=.99 c$ with collisions for $\left(\nu=0,0.2 m_{D}, 0.5 m_{D}, 0.8 m_{D}\right.$ (top to bottom panel). Right panel: These plots show the corresponding equipotential lines. 